\title{
Present problems of vibration isolation in heavy mining machines at long-term cyclic loads
}

\author{
Vitalii Dyrda ${ }^{1, *}$, Anatolii Kobets ${ }^{2}$, Ievgeniia Bulat $^{3}$, Serhii Slobodian ${ }^{4}$, Mykola Lysytsia ${ }^{1}$, \\ Serhii Sokol ${ }^{2}$, Vladimir Lapin ${ }^{5}$, and Hennadii Ahaltsov ${ }^{1}$ \\ ${ }^{1}$ Institute of Geotechnical Mechanics named by N. Poljakov of National Academy of Sciences of \\ Ukraine, 49005, Dnipro, Simferopolska Str., 2a, Ukraine \\ ${ }^{2}$ Dnipro State Agrarian and Economic University, 49600, Dnipro, Serhiia Yefremova Str., 25, \\ Ukraine \\ ${ }^{3}$ Prydniprovsky Scientific Center of National Academy of Sciences of Ukraine and of Ministry of \\ Education and Science of Ukraine, 49600, Dnipro, Leshko-Popelia Str., 15, Ukraine \\ ${ }^{4}$ State Agrarian and Engineering University in Podilya, 32300, Kamianets-Podilskyi, Khmelnytskyi \\ region, Schevchenko Str., 13, Ukraine \\ ${ }^{5}$ Kazakh Research and Design Institute of Construction and Architecture, 050036, Almaty, 3rd \\ microdistrict, 45/1, Kazakhstan
}

\begin{abstract}
On the basis of the developed simulation model and the Boltzmann-Volterra integral relations with kernels of relaxation and aftereffect, an equation was worked out, which made it possible to take into account the rubber viscoelastic properties in full volume; in this equation, stiffness operator of elastic suspension in the machine is written by using fractional exponential function of the Yu. Rabotnov's type; on the basis of the mathematical model, the basic parameters of the machine under the study were calculated; in particular, for the vortex mixer, the time dependences of amplitude of the mixer housing vibrations and coefficient of vibration isolation efficiency were calculated with taking into account aging of elastic link material in the machines; the calculation results were compared with the results of industrial tests of the vortex mixer operation lasting for 16 years. The theory and method for calculating vibration isolation systems with rubber elastic links for heavy mining machines were developed with taking into account material structure changes due to the effects of aging.
\end{abstract}

\section{Introduction}

Vibration isolation of machines is part of general theory of elastic system vibrations. A great volume of literature is available on this issue; it is enough to mention the fundamental monographs of S.P. Tymoshenko, Den Gartog, Ya.G. Panovko, Ye.S. Sorokin, G.S. Pisarenko, V.L. Biderman, E.E. Lavendel and a number of other authors in order to present the importance of this problem for mechanical engineering and existing comprehensive knowledge about it. For some systems and facilities of vibration isolation, state standards have been worked out. However, the problem of protecting operators,

*Corresponding author: vita.igtm@gmail.com 
machines, instruments, equipment, buildings and structures against the effects of vibrations and sound pressure is still relevant.

This issue is especially important for such complex technical systems as mining-andprocessing enterprises, construction and agricultural industries, where heavy equipment is installed in a certain sequence depending on technological requirements; besides, sometimes machines are installed in three-dimensional space: for example, mixers can be placed on floors at the elevation of $+18 \mathrm{~m}$, etc.

At the same time, vibrational load on the equipment is significant, and frequency spectrum includes the entire range from low to high. Oscillations most often occur randomly, such as white noise, and, as a rough approximation, they can be considered as quasi-harmonic. High frequencies are usually suppressed with the help of damping pads: the higher is vibration frequency, the easier it is suppressed.

For suppressing low frequencies, special vibration isolation systems are used. Usually, it is vibration isolators, dampers, dynamic dampers, etc., which are installed between the machine and frame (foundation, supporting structure). The many-year practice of operating the machines shows that the most effective vibration isolation systems are those that use rubber vibration isolators. With correctly chosen parameters and design of the rubber elements, such systems provide effective protection against vibrations and sound pressure, contribute to the intensification of technological process and increase productivity.

In order to create an effective vibration isolation system, especially low-frequency, it is necessary to satisfy rather contradictory requirements: vibration isolators in heavy machines should feature high bearing capacity and low rigidity. The use of rigid elastic suspensions makes it possible to suppress high frequencies effectively enough, while low frequencies, which are the most dangerous, are suppressed poorly; moreover, such oscillation mode is possible when excitation frequency is close to or coincides with natural frequency of the system (the phenomenon of resonance), which is extremely undesirable because under the action of random excitations, which are usual phenomenon in any technological machine, vibration of machine may be increased making the vibration isolation system ineffective. Therefore, soft vibration isolation system is more preferable, though it requires a special design of vibration isolators and choosing of materials with appropriate stiffness and dissipative properties. Soft system allows suppressing the entire frequency spectrum, both high and low frequencies. Its disadvantages include possible rocking of machine in horizontal position; to escape this undesirable effect, resistant vibration isolators are usually used.

Below are described some of the issues of vibration isolation in heavy mining and processing machines operating in extreme conditions. During the operation, such machines experience stationary dynamic loads, effect of aggressive medium created by material being processed and significant temperature differences. Such machines include vortex mixers, pelletizers-mixers, crushers, vibratory feeders, screens, etc. In all of these machines, vibration leads to increased dynamic stresses in parts and assemblies, higher sound pressure, deterioration of technology of material processing and worse quality of original product, and intensive wear of parts. Besides, vibration adversely affects structural units of the sinter plants and is often the main cause of the destruction of foundations.

Usually, rubbers of special design are used as elements of elastic suspension in the heavy mining machines. Today, rubber in vibration isolating systems of heavy mining and processing machines cannot be replaced by any other of the existing materials due to its feature of large deformation reversibility, high elastic and dissipative properties, and absence of failure suddenness; besides, its fatigue characteristics exceed fatigue of any of existing structural materials. The rubber disadvantages include dependence of viscoelastic properties on the loading mode, aggressive medium and duration of operation.

The purpose of this work is to develop a theory of vibration isolation in the heavy 
mining machines with taking into account effects of aging (i.e. changes in the material structure during operation) of their elastic links and the confirmation of the analytical model by the results of lengthy industrial tests.

\section{Method}

\subsection{Simulation models}

In most cases, such models (phenomenological, synergistic, and others) are based on the methods of taking energy dissipation into account (inelastic resistances, in other words) by using the Kelvin-Focht, Maxwell and other viscoelastic models. However, rubber does not comply with the simplified Kelvin-Focht models, and its dissipative properties are not directly proportional to the strain rate, i.e. its dissipation factor $\psi$ is not proportional to the increase of the angular frequency of loading $\omega$. Results of numerous experimental studies indicate [1] that, for rubber, the curve $\psi(\omega)$ in a wide frequency range feature rather complex configuration with different relaxation peaks. In Figure 1, such a curve for lowfilled rubber is shown, which was built by the device "Instron", model 1126, at $293 \mathrm{~K}$ [1]. As it can be seen, proportionality of the energy losses of the strain rate is observed exclusively in certain sections of the frequency curve and within the narrow frequency range. In the frequency range, which is characteristic for the vibration isolation systems of heavy machines (the shaded area in Figure 1), with an increase of the deformation rate, the energy dissipation decreases, and this fact contradicts the Kelvin-Focht and Maxwell models.

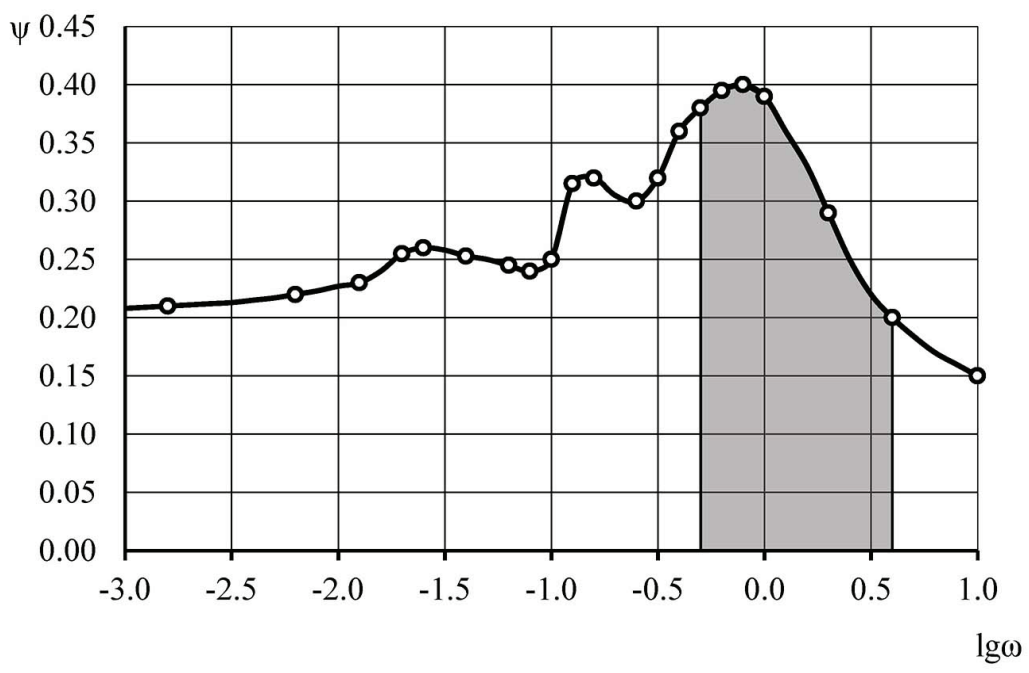

Fig. 1. Frequency dependences of the energy dissipation factor for rubber with the filling of 5.0 mass parts of carbon black.

Nevertheless, in the theory of oscillations, these models are used in most cases due to the well-developed mathematical apparatus and minimum number of information parameters in the equations. This leads to some errors, especially in the field of resonance. More appropriate is the use of integral equations of the Boltzmann-Volterra type with relaxation and aftereffect kernels. However, this requires knowledge of rheological characteristics of the material, which is associated with additional experimental studies of vibration isolators.

Such famous scientists as V.M. Poturaev, V.P. Franchuk, A.H. Chervonenko and many 
other authors in their researches of dynamics of mining vibration machines (screens, feeders, conveyors, etc.) took into account energy dissipation by using of the Kelvin-Focht and Maxwell models. In the works of modern authors, the same models were used in the study of dynamics of vibration machines [2-7].

In all these researches, as well as in the works of other authors, only initial values of the machine parameters were taken into account, while their in-time changes due to the effects of the elastic link aging were not considered.

However, consideration of such effects is of great importance; the problem arose due to the need to create vibration isolation systems with a long service life (approximately $15-20$ years) and with high degree of reliability. Solution of the problem lies in considering effects of rubber aging in mathematical models, i.e. in-time instability of stiffness and dissipative characteristics during the operation of vibration isolation system. The time characteristics of the rubber mechanical properties (mainly informational parameters: shear modulus $G$ and dissipation factor $\psi$ ) can be obtained either by long-term experimental studies or by heat aging according to existing standards. The obtained aging functions $G(t)$ and $\psi(t)$ can be included directly into the equations for the machine dynamics.

The theory of vibration isolation in heavy mining machines with taking into account intime instability of main informational parameters of elastic link material is described below.

\subsection{Mathematical models}

In general, kinematic scheme of most of the mining vibration machines can be represented as a single-mass system with an elastic-viscous suspension and one degree of freedom. Let's consider two methods used for calculating such systems.

The first method assumes that mechanical reaction of elastic suspension in machines is described by the Kelvin-Focht model, and stress obeys the law

$$
\sigma=E \varepsilon+\mu E \dot{\varepsilon},
$$

where $E$ is modulus of elasticity, $\mathrm{N} / \mathrm{m}^{2} ; \varepsilon$ is relative deformation of the vibration isolator; $\mu$ is coefficient of the rubber internal resistance, $\mathrm{s}$; value of which is proportional to the strain rate; for medium filled rubbers $\mu=2 \cdot 10^{-3} \mathrm{~s}$ (the value was obtained experimentally in the research works of V.M. Poturaev).

In this case, the equation of motion of the mass $m$ can be written as

$$
\ddot{y}+\mu \omega_{0}^{2} \dot{y}+\omega_{0}^{2} y=q_{1} \sin \omega t .
$$

where $y$ is coordinate.

For the amplitude of stationary forced oscillations, the following ratio is correct

$$
A_{\omega}=\frac{q_{1}}{\sqrt{\left(\omega_{0}^{2}-\omega^{2}\right)^{2}+\mu^{2} \omega^{2} \omega_{0}^{4}}},
$$

where $\omega$ is angular frequency of the system forced oscillations, $\mathrm{rad} / \mathrm{s} ; \omega_{0}$ is natural angular frequency of the system, $\mathrm{rad} / \mathrm{s} ; q_{1}$ is inertia force per unit of oscillating mass $\mathrm{N} / \mathrm{kg}$.

The second method assumes that mechanical reaction of rubber is described by integral relation of the Boltzmann-Volterra type with relaxation and aftereffect kernels. In this case, the equation (2) in the operator form will be rewritten as

$$
m \ddot{y}+C_{t} y=Q \sin \omega t
$$


where $m$ is the mass of the system, $\mathrm{kg} ; C_{t}$ is the stiffness operator of the elastic suspension, $\mathrm{N} / \mathrm{m} ; Q$ is the force of inertia, $\mathrm{N}$.

$$
\begin{gathered}
C_{t}=C_{0}\left[1-\mathfrak{R}_{\alpha}^{*}(-\beta)\right], \\
\mathfrak{R}_{\alpha}^{*}(-\beta) \varepsilon(t)=\int_{0}^{t} \mathfrak{R}_{\alpha}(-\beta, t-\tau) \varepsilon(\tau) d \tau, \\
\mathfrak{R}_{\alpha}(-\beta, t-\tau)=\chi(t-\tau)^{\alpha} \sum_{n=0}^{\infty} \frac{(-\beta)^{n}(t-\tau)^{n(1+\alpha)}}{\Gamma[(n+1)(1+\alpha)]},
\end{gathered}
$$

where $C_{0}$ is instantaneous value of elastic suspension stiffness, $\mathrm{N} / \mathrm{m} ; \mathfrak{R}_{\alpha}(-\beta, t-\tau)$ is exponential function of fractional order of the Yu. Rabotnov's type; $\alpha, \beta, \lambda, \chi$ are rheological parameters of rubber; $\Gamma$ is gamma function.

For determining rheological parameters of rubber, the following relations were obtained

$$
\begin{gathered}
\psi=2 \pi B(\omega) ; \\
\frac{G(\omega)}{G_{0}}=1-A(\omega) ; \\
A(\omega)=\frac{\chi\left(\omega^{1+\alpha} \cos \delta+\beta\right)}{\omega^{2(1+\alpha)}+2 \omega^{1+\alpha} \beta \cos \delta+\beta^{2}} ; \\
B(\omega)=\frac{\chi \omega^{1+\alpha} \sin \delta}{\omega^{2(1+\alpha)}+2 \omega^{1+\alpha} \beta \cos \delta+\beta^{2}} ; \\
\lambda=\frac{G_{0}-G_{\infty}}{G_{0}} ; \quad \alpha=1-\frac{4}{\pi} \arctan \frac{\psi_{\max }}{\pi \lambda} ; \\
t_{0}=\left[\omega\left(\psi_{\max }\right)\right]^{-1} ; \quad \beta=\frac{1}{t_{0}^{1+\alpha}} ; \quad \chi=\frac{\lambda}{t_{0}^{1+\alpha}}
\end{gathered}
$$

where $\psi$ is energy dissipation factor; $\psi_{\max }$ is the maximum dissipation factor; $A(\omega)$ and $B(\omega)$ are rheological characteristics of rubber (the Fourier sine and cosine transformation of the fractional exponential function); $G(\omega)$ is current value of the shear modulus, N/m $\mathrm{m}^{2} ; G_{0}$ is instantaneous value of the shear modulus, $\mathrm{N} / \mathrm{m}^{2} ; G_{\infty}$ is conditionally-equilibrium shear modulus, $\mathrm{N} / \mathrm{m}^{2} ; t_{0}$ is generalized time of relaxation, $\mathrm{s}$.

For medium filled rubber of the 2959 type used below, rheological parameters have the following values

$$
\alpha=-0.60 ; \quad \beta=1.1 ; \quad \lambda=0.58
$$

With taking into account the above ratios, the expression of the oscillation amplitude has the following form

$$
A_{\omega}=\frac{Q}{m \sqrt{\left\{\omega_{00}^{2}[1-A(\omega)]-\omega^{2}\right\}^{2}+B^{2}(\omega) \omega_{00}^{4}}},
$$

where $\omega_{00}$ is natural angular vibration frequency of an ideally elastic system, $\mathrm{rad} / \mathrm{s}$.

The expression (14) makes it possible to determine more accurately the amplitudefrequency and phase characteristics under transient conditions, for example, in the resonance region, i.e. when $\omega=\omega_{0}$; when $\omega / \omega_{0}>\sqrt{2}$ the expressions (3) and (14) give 
approximately the same results.

As it can be seen, a mathematical model with usage of the Volterra integral relations is based on more rigorous assumptions and considers more accurately the rubber viscoelastic properties than the Kelvin-Focht model. It is most effective for studying nonlinear systems, transient processes in oscillatory systems, as well as the systems, whose rheological characteristics substantially depend on time, loading mode or slowly changing temperature (external temperature or dissipative self-heating temperature).

\subsection{Evaluation of vibration isolation system effectiveness}

Equations (1) and (3) allow obtaining expressions for the basic parameters of vibration isolation system in the following form [1] for coefficient of vibration isolation

$$
\eta=\frac{\sqrt{1+Z^{2} \frac{4 \psi^{2}}{16 \pi^{2}+\psi^{2}}}}{\sqrt{\left(1-Z^{2}\right)^{2}+Z^{2} \frac{4 \psi^{2}}{16 \pi^{2}+\psi^{2}}}} ; \quad Z=\frac{\omega}{\omega_{0}} ; \quad \omega_{0}=\sqrt{\frac{C}{m}},
$$

where $C$ is elastic suspension stiffness, N/m. In this formula, dissipation factor $\psi$ is determined either experimentally or by the known rubber rheological parameters $\alpha, \beta, \lambda$ calculated by formula (8), i.e. $\psi=2 \pi B(\omega)$. In this case, formula (15) takes the following form

$$
\eta=\frac{\sqrt{\left(1+Z^{2}\right)^{2} \frac{16 \pi^{2} B^{2}(\omega)}{16 \pi^{2}+4 \pi^{2} B^{2}(\omega)}}}{\sqrt{\left(1-Z^{2}\right)^{2}+Z^{2} \frac{16 \pi^{2} B^{2}(\omega)}{16 \pi^{2}+4 \pi^{2} B^{2}(\omega)}}}
$$

Effectiveness of vibration isolation is equal to

$$
\mathfrak{I}=(1-\eta) \cdot 100 \%
$$

Depreciation coefficient (or dynamic coefficient)

$$
K_{a}=\sqrt{\left(1-Z^{2}\right)^{2}+\psi^{2} Z^{2}}
$$

or, with taking into account $\psi=2 \pi B(\omega)$

$$
K_{a}=\sqrt{\left(1-Z^{2}\right)^{2}+4 \pi^{2} B^{2}(\omega) Z^{2}} .
$$

From formulas (16) and (17), very important conclusions are follow:

- when $Z<1$, vibration isolation coefficient $\eta$ is close to 1 , and vibration isolation system has almost no effect on the process of the machine oscillations;

- at $Z=1$, the system operates in the resonance region;

- when $Z \geq \sqrt{2}$, vibration isolation system becomes effective; coefficient of vibration isolation decreases with decrease of the system damping; therefore, value for the relative damping is usually chosen so that, on the one hand, to obtain a small increase of the elastic system force transmission, especially in the resonance region, and, on the other hand, 
vibration isolation should be satisfactory in case of higher frequencies;

- when $Z \geq 5$, vibration isolation efficiency is $\eta=1$; this indicates that possibilities of the elastic system are almost exhausted and there is no point in doing it softer; therefore, in practice, $Z=2.5 \ldots .5 .5$ is usually used;

- in some technological machines, irregular shocks occur, which can increase amplitude of oscillations being a source of additional dynamic forces. Usually, in such cases, vibration dampers are made of well-damping materials such as filled rubber, which, of course, increases energy dissipation, but, at the same time, increases stiffness of elastic suspension and, therefore, reduces coefficient of vibration isolation in the high-frequency region. It is known that high frequencies are better suppressed by vibration isolation system than low frequencies. As it is mentioned above, vibration isolators with low stiffness and high loadcarrying capacity are used exactly for reducing the low-frequency vibrations. Vibration isolators with low stiffness in the direction of basic oscillations are the most effective; however, in this case, an effect of the system rocking in the horizontal direction occurs. To eliminate this undesirable effect, additional vibration isolators-limiters, or so-called resistant vibration isolators are usually used;

- it should be noted that at starting and stopping, any machine passes the resonance region, and amplitude of oscillations increases; usually, the use of rubber vibration isolators significantly reduces vibrations at resonance;

- it is assumed that the vibration isolation system is installed on absolutely rigid base (floors, foundation) with the mass $m_{1}$, which does not demonstrate any vibration displacement during the machine operation. In practice, this assumption is not always true, and the floor, for example, can vibrate with some frequency. Here, it is very important to choose parameters for the vibration isolation system in such a way as to avoid highly undesirable resonance. For such systems, the resonant frequency is determined by the ratio

$$
f_{0}^{\prime}=f_{0} \sqrt{1+\frac{m}{m_{1}}},
$$

where $f_{0}$ is resonant frequency of the system without taking into account reaction of the foundation, $\mathrm{Hz} ; f_{0}^{\prime}$ is resonant frequency of the system with taking into account reaction of the foundation, for example, floor, $\mathrm{Hz}$.

As it can be seen, the task of choosing the right parameters for the vibration isolation system is reduced to the problem of choosing vibration isolators with optimal stiffness and damping properties and keeping the frequency ratio within the range of $Z=2.5 \ldots .5$; in this case, effectiveness of vibration isolation is always $\mathfrak{I} \cong 80 \%$.

Equations (2) and (4) or their simplified variants are most widely used in engineering practice when calculating the systems of vibration isolation for the machines and structures. Analysis of energy dissipation included into these equations indicates the following:

- with elliptical hysteresis loop (linear stiffness characteristic of elastic system), energy dissipation practically does not change natural frequency of the system oscillations;

- configuration of the hysteresis loop has little effect on the value of the system oscillation amplitude; it affects the frequency and position of the resonant peak relative to the vertical $\omega / \omega_{0}=1$;

- in the existing mathematical models of elastic system oscillations, method of energy dissipation accounting makes it possible to reveal its influence only in the region of resonance; with stationary oscillations, the effect of energy dissipation is insignificant.

All these considerations should be considered when calculating a concrete system of vibration isolation for the machines. 


\subsection{Experimental studies of the system of vibration isolation of vortex mixers}

A set of experimental studies of vortex mixers installed on a monolithic reinforced concrete slab at the level of $+10.800 \mathrm{~m}$ in the mixing section of the sintering plant of the Northern Mining and Processing Plant (Kryvyi Rih) was carried out with the help of the Brüel \& Kjær measuring equipment. A design feature of the reinforced concrete floors is that one of its sides at the discharge part of the mixer is free and rests on steel racks.

The mixer is a hollow metal cylinder (Fig. 2), in which rotor with special blades for mixing the processed raw materials revolves; the rotor is actuated by electric drive. Due to the imperfections of manufacture of elements for the mixer, inaccuracies of their assembly and specificity of the mixing process technology (sticking of the product on the rotor blades, the material falling from the blades according to a random law, etc.), a strict circular symmetry of the mixer elements moving relative to the axis of rotation is disturbed and leads to occurrence of centrifugal forces, dynamic imbalance and vibrations with a broadband spectrum of disturbing forces, which are transmitted to the reinforced concrete floors.

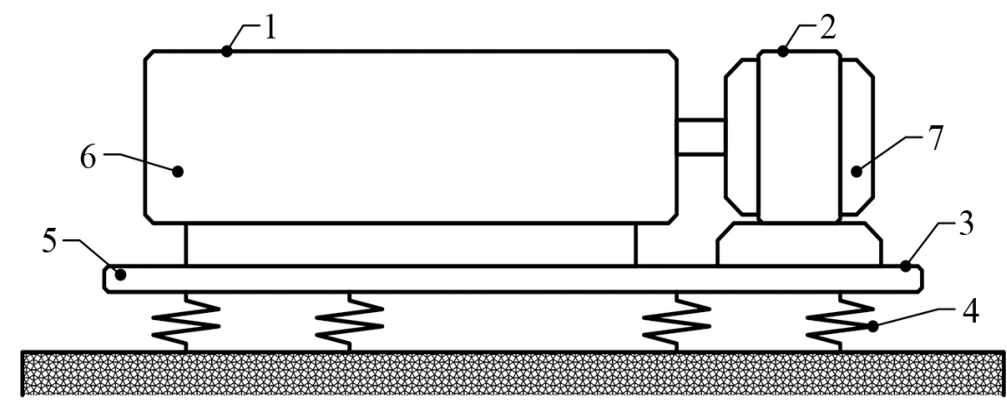

Fig. 2. Mixer scheme: 1 -drum; 2 -drive; 3 -frame; 4-vibration isolators; 5, 6, 7 -points of vibration measurement.

This dynamic imbalance, along with the rotation symmetry of the moving parts during the mixer operation, increases with operating time due to the wear of its elements. When repairing the mixer, some disadvantages are eliminated by way of replacing the rotor blades, removing stuck material from the rotor, etc. However, it is not possible to completely eliminate this constructive and technological imbalance.

For the mixer rigidly mounted on the reinforced concrete floor, the following values of the vibration parameters were experimentally obtained: oscillation amplitude of the mixer supporting structure was $0.22 \mathrm{~mm}$, oscillation frequency was $14 \mathrm{~Hz}$; amplitude of oscillations of the housing of the main mixer assemblies was $(0.11-0.17) \mathrm{mm}$ in the vertical plane and $(0.05-0.11) \mathrm{mm}$ in the horizontal plane; amplitude of oscillations of the floors between the mixers was $(0.17-0.21) \mathrm{mm}$ in the vertical plane and $(0.22-0.27) \mathrm{mm}$ in the horizontal; sound pressure level was $110 \mathrm{~dB}$; level of vibrations and sound pressure exceeded sanitary standards by about 1.5 times. It was also noted that vibrations led to intensive wear of the mixer elements and destruction of the floor.

After the mixer was installed on the rubber vibration isolators, the experimental studies showed the following: oscillation amplitude of the mixer supporting structure was $0.07 \mathrm{~mm}$; sound pressure level was $80 \mathrm{~dB}$; dynamic loads on the main elements of the mixer decreased by three times; effectiveness of the vibration isolation system for reducing the vibrations transmitted to the floor was $85 \%$; level of the floor vibration and sound pressure were reduced up to sanitary standards.

The vibration isolation system remained effective for about 9 years. Due to the aging of rubber, vertical stiffness of rubber vibration isolators increased on average by $60 \%$, energy 
dissipation factor decreased by 4 times. The consequence was that stiffness and dissipative characteristics of the vibration-isolation system exceeded permissible limits for the parameter changing, and the system of vibration isolation became ineffective.

During the operation, as a result of aging of rubber elements in the vibration isolation system, amplitude of oscillations of the floor increased by almost 9 times (from the initial value $A_{\omega}=0.027 \mathrm{~mm}$ to $A_{\omega}=0.21 \mathrm{~mm}$ ).

\subsection{Calculation of vibration isolation system of the mixer with taking into account the rubber aging}

Data for calculation:

- mass of the vibration isolation system is $m=35000 \mathrm{~kg}$;

- frequency of forced oscillations is $-14 \mathrm{~Hz}$;

- number of vibration isolators: 26 supporting isolators and 8 stop isolators;

- static load on one supporting vibration isolator is $15.0 \mathrm{kN}$;

- metal-rubber vibration isolators with rubber element dimensions: diameter $180 \mathrm{~mm}$, height $100 \mathrm{~mm}$;

- rubber 2959 with the following initial physical and mechanical characteristics: conditionally-equilibrium modulus of elasticity is $E_{g}=3.7 \mathrm{MN} / \mathrm{m}^{2}$; dynamic (at the frequency of forced oscillations of the mixer $14 \mathrm{~Hz}$ ) elastic modulus is $E_{0}=4.8 \mathrm{MN} / \mathrm{m}^{2}$; energy dissipation factor is $\psi=0.35$.

In total, the vibration isolation system was monitored for 16 years: from time to time, vibration isolators (not less than three) were taken out from the system and checked by a complex of mechanical tests. The test results are shown in Figure 3 and Figure 4.

As it can be seen, dynamic modulus of elasticity was changed by exponential law; functional dependence $E^{0}(t)$ can be represented as

$$
E^{0}(t)=E_{1}^{0}+\left(E_{2}^{0}-E_{1}^{0}\right) \exp \left(k_{1} t\right)
$$

where $E_{1}^{0}$ and $E_{2}^{0}$ are initial and final values of dynamic modulus of elasticity, respectively, $\mathrm{N} / \mathrm{m}^{2} ; k_{1}$ is the rate constant.

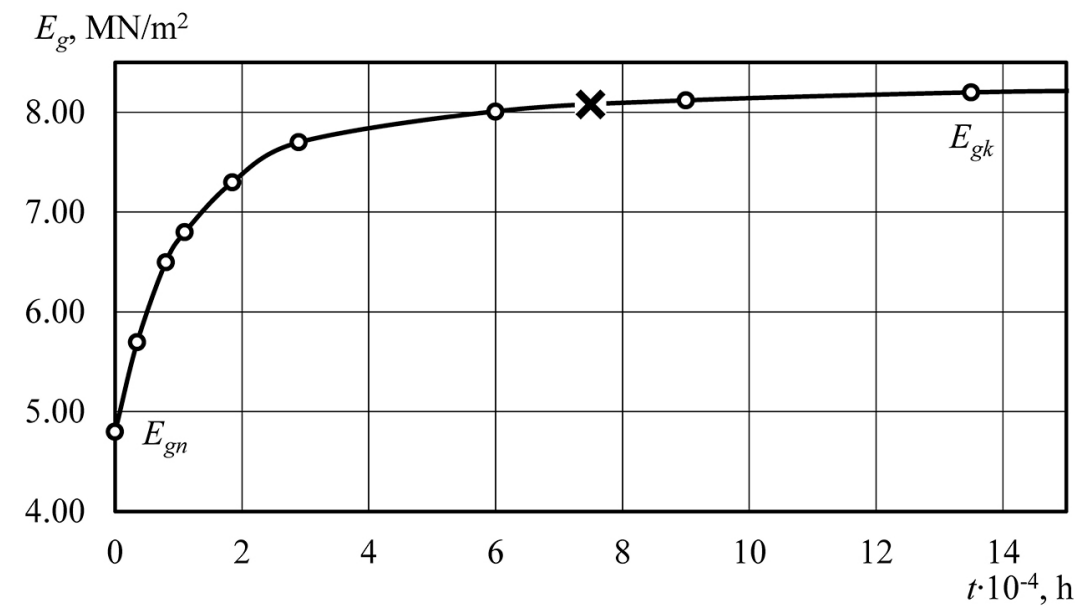

Fig. 3. Time dependence of dynamic modulus of compression; $E_{g n}, E_{g k}$ are initial and final values of the conditionally-equilibrium modulus of elasticity, $x$-lower limit of the vibration isolator service life. 


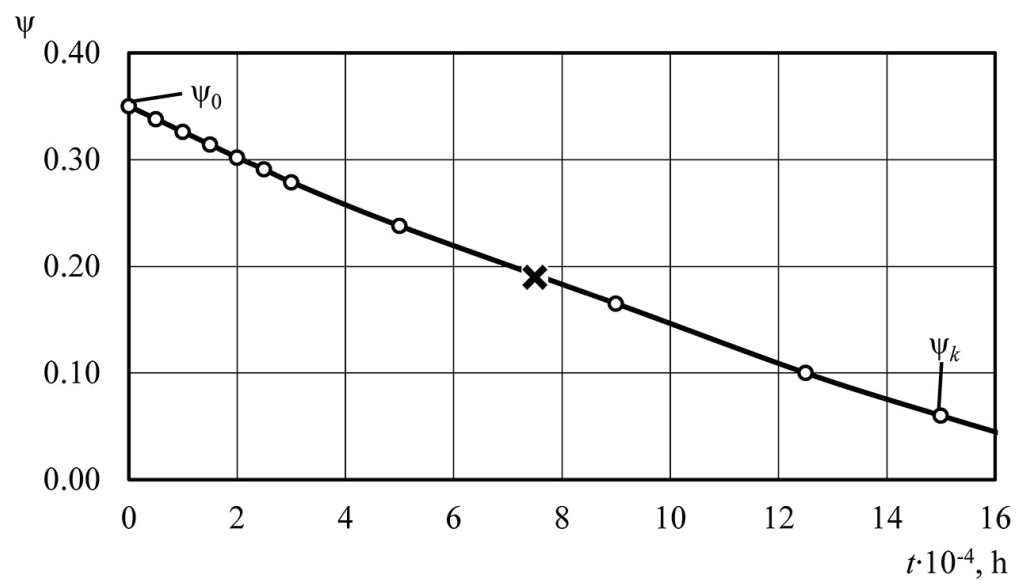

Fig. 4. Time dependence of energy absorption coefficient; $\psi_{0}$ and $\psi_{k}$ are initial and final values of energy dissipation factor, $x$ - lower limit of the vibration isolator service life.

The dependence $\psi(t)$ can be described by the relation

$$
\psi(t)=\psi_{0}-k_{2} t
$$

where $\psi_{0}$ is initial value of the absorption coefficient; $k_{2}$ is the rate constant, $\mathrm{s}^{-1}$.

By putting relations (21) and (22) into equations (3) and (14), we obtain the time dependences of the mixer oscillation amplitude (Fig. 5) and coefficient of vibration isolation (Fig. 6).

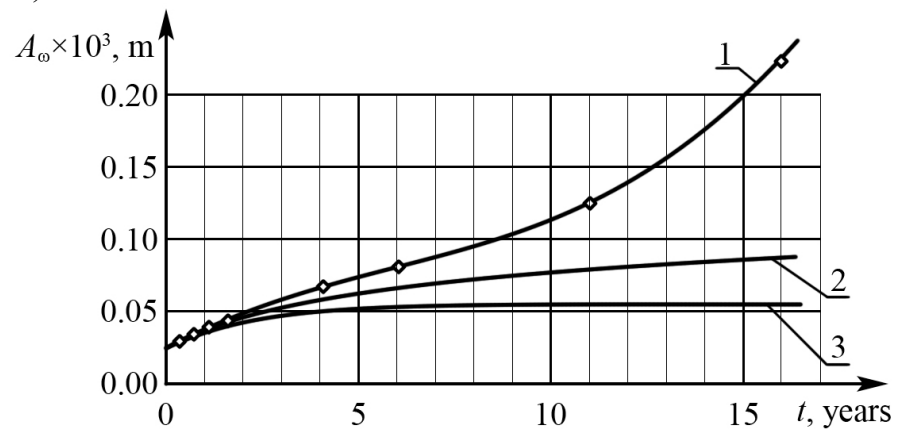

Fig. 5. Time dependence of amplitude of the mixer housing vibration: 1 - experimental curve with taking into account wear of the mixer elements; 2 - calculation by equation (4); 3 - calculation by equation (2).

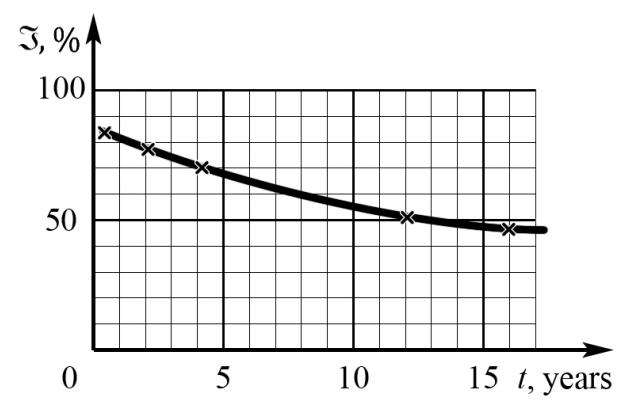

Fig. 6. Time dependence of coefficient of vibration isolation effectiveness. 
For the considered vibration isolators: $\psi_{0}=0.35 ; k_{2}=0.085 \cdot 10^{-8} \mathrm{~s}^{-1} ; E_{1}^{0}=4.80 \mathrm{MN} / \mathrm{m}^{2}$; $E_{2}^{0}=8.29 \mathrm{MN} / \mathrm{m}^{2} ; k_{1}=1.1 \cdot 10^{-5} \mathrm{~h}^{-1}$.

It should be mentioned that during the operation of the vibration isolators ( $t=16$ years), elastic modulus increased by $60 \%$, and energy dissipation rate decreased by more than $400 \%$, which, in general, indicates a change in the structure of the material and its relaxation properties and decrease of its ability to absorb energy of mechanical vibrations.

In practice, such changes in the rubber basic parameters led to the following: the vibration isolation system remained effective for about 9-10 years (about 70-85 thousand hours); during further operation, parameters of the rubber exceeded the limits of permissible values, and the vibration isolation system lost its functionality; amplitude of the mixer housing oscillations exceeded sanitary norms.

Calculation of elastic characteristics of the rubber vibration isolators is considered in [8].

\section{Discussion of the results}

1. Despite diversity of methods for accounting energy dissipation during vibrations of mechanical systems, modern mathematical models, as a whole, do not consider viscoelastic effects accompanying the rubber deformation in full volume. When constructing the amplitude characteristic of the system (Fig. 5) according to the equations (14) and (16) and determining the coefficient of vibration isolation by formulas (18) and (19), values of $A$ and $\eta$ depend on the energy dissipation factor $\psi$. However, when considering rubber aging effect on the dynamics of the mixer, changes of stiffness characteristics play a more important role since they determine natural frequency of the system oscillation.

2. The increase of dynamic stiffness (Fig. 5, curve 3) of the system elastic suspension by $60 \%$ (during 16 years of operation) gives a calculated increase of amplitude of oscillations by $(20-25) \%$ according to equation (2) and (21); equation (14) more accurately takes into account the viscoelastic effects (Fig. 5, curve 2), but coincidence with the experiment (Fig. 5, curve 1) is observed exclusively in the first $3-4$ years of operation. Such significant discrepancies between theoretical and experimental curves are explained by the following circumstances: in the calculations, value of the inertia force per unit mass of the mixer was assumed to be constant. However, for most of the mining and smelting machines, during their operation, it is not only the parameters of elastic suspension that change; due to the wear of moving elements, degree of their imbalance also changes and leads to increase of inertial force per unit mass. Of course, elimination of wear results in decrease of this force, however, it can significantly increase during the overhaul period (for example, due to sticking of concentrate to the blades, uneven wear of the blades, wear of gearbox parts, etc.).

All this, as follows from Figure 5, should be taken into account when designing and calculating the vibration isolation systems, which should be made with a certain reserve allowing to be within the limits of permissible values for a certain period of time even at some deviations from the set parameters of the mixer (for example, changed mass or disturbing force). To this end, dynamic factor should be within the range of 4.5-5.0. The considered vibration isolation system of the mixer was designed exactly in this way, and even when stiffness of vibration isolators and disturbing force (due to the wear of the mixer elements) were changed (stiffness was changed by $60 \%$ ), it still remained within the limits of sanitary standards and technological requirements for $9-10$ years.

3. Energy dissipation affects amplitude of the mixer oscillations; it affects frequency and position of the resonant peak relative to the vertical $\omega / \omega_{0}=1$. Rubber aging increases the amplitude of the mixer oscillations (Fig. 5), changes the system's natural oscillation 
frequency and shifts the value of $\omega / \omega_{0}$, i.e. position of the resonance peak.

4. Energy dissipation affects the coefficient of vibration isolation $\eta$; rubber aging significantly increases $\eta$ mainly due to the changed frequency ratio $\omega / \omega_{0}$; and efficiency of the vibration isolation decreases accordingly (Fig. 6).

\section{Conclusions}

1. Any vibration isolation system in the machines operating under dynamic loads for a long period of time should be calculated with taking into account the rubber aging and inevitable disturbing force increase with time.

2. The theory and method are developed for calculating the vibration isolation systems with rubber elastic links for heavy mining machines, whose information parameters change with operating time due to the aging of the material.

3. According to the accepted mathematical models, energy dissipation of the machine elastic links affects the in-time changes of vibration amplitude and coefficient of vibration isolation; in addition, it significantly reduces noise, hence, improving safety of operators' work and machine operation.

\section{References}

1. Bulat, A.F., Dyrda, V.I., Zvyagilskiy, Ye.L. (2016). Elastomers in mining. Naukova dumka, Kyiv, Ukraine

2. Zabolotny, K.S., Sirchenko, A.A., Zhupiev, A.L. (2016). Design and analysis of the oscillating system's of self-propelled feeding bin BPS-940. Vibration in engineering and technology, 2 (82), 16-21, http://vibrojournal.vsau.org/files/pdfa/3011.pdf

3. Yaroshevich, N.P., Zabrodets, I.P., Yaroshevich, T.S. (2015). Dynamics of vibrating machines starting with unbalanced drive in case of bearing body flat vibrations. Scientific Bulletin of National Mining University, 3, 39-45

4. Ilin, S.R., Samusia, V.I., Ilina, I.S., Ilina, S.S. (2016). Influence of dynamic processes in mine hoists on safety of exploitation of shafts with broken geometry. Scientific Bulletin of National Mining University, 3, 48-53

5. V. Dyrda, A. Kobets, Ie. Bulat, V. Lapin, N. Lysytsia, H. Ahaltsov, S. Sokol. Vibroseismic protection of heavy mining machines, buildings and structures. E3S Web of Conferences, International Conference Essays of Mining Science and Practice, 109 (2019). https://doi.org/10.1051/e3sconf/201910900022

6. V. Dyrda, A. Kobets, V. Pukhalskyi, Yu. Kozub, O. Chernii, Dynamics of vibratory partitioned feeders for the uranium ore drawing and feeding. E3S Web of Conferences, International Conference Essays of Mining Science and Practice, 109 (2019). https://doi.org/10.1051/e3sconf/201910900023

7. O. Voloshyn, O. Riabtsev. Some important aspects of rock mechanics and geomechanics. E3S Web of Conferences, International Conference Essays of Mining Science and Practice, 109 (2019). https://doi.org/10.1051/e3sconf/201910900114

8. Bulat, A.F., Dyrda, V.I., Lysytsya, M.I., Grebenyuk, S.M. (2018). Numerical Simulation of the Stress-Strain State of Thin-Layer Rubber-Metal Vibration Absorber Elements Under Nonlinear Deformation. Strength of Materials, 3 (50), 387-395, https://doi.org/10.1007/s11223-018-9982-9 